\title{
BREEDING FOR CREEPING ROOT IN ALFALFA (MEDICAGO MEDIA PERS.) ${ }^{1}$
}

\author{
F. H. W. MorLey ${ }^{2}$ and D. H. Hernrichs ${ }^{3}$ \\ [Received for publication October 23, 1959]
}

\begin{abstract}
An alfalfa population, consisting of progenies from crosses among 54 plants which had been selected out of a large population of Medicago falcata L. $\mathrm{x}$ Medicago media Pers. origin, was scored for degree of creeping-root development. These scores were statistically analysed on the basis of full-sib and half-sib groups. The estimate of $\operatorname{Cov}(\mathrm{FS})-\operatorname{Cov}(\mathrm{HS})$ was 0.062 , and Cov(HS) was 0.091. Therefore, it was concluded that genotypic variation was predominantly additive.

Mass selection alone is predicted to be more efficient in breeding for the creeping-rooted character in alfalfa than a combination of mass selection and progeny testing. However, since other characters will usually be selected for along with the creeping-rooted character, the progeny test may have to be used. If progeny testing is used, the optimum number of progeny per parent under test should be approximately 30 , provided these have been obtained from at least 8 to 10 different, unrelated, and randomlyselected mates.
\end{abstract}

\section{INTRODUCTION}

A description of the creeping-rooted character in alfalfa and its potential agronomic value has been presented by Heinrichs (2) and Murray (8). Recently the variety Rambler, within which 65 per cent of the plants are creeping rooted, has been released in Canada and is at present undergoing extensive tests (3).

Alfalfa breeders in many countries are keenly interested in the possibility of incorporating the creeping-rooted character into strains adapted to local conditions. It is, therefore, of wide interest to obtain information on the inheritance of this trait, and to use the information in the design and evaluation of breeding programs.

In this paper, data obtained on the creeping-rooted character from a large population of alfalfa grown in experimental blocks at Swift Current, Saskatchewan, have been statistically analysed with the object of determining whether genotypic variance in the population was predominantly additive or included non-additive components. If non-additive genotypic variance should be important, special techniques of selection, which make use of specific combining ability, would be necessary. However, if additive genotypic variance is large relative to other sources of variance, mass selection and other techniques of selection for general combining ability would be preferred. The relative emphasis to be placed on performance, progeny testing or other aids to selection depends on resources demanded by various breeding plans as well as on the different components of variance.

On the basis of the results obtained several breeding plans were examined with the object of demonstrating some of the principles used in applying the results of the analysis. It is possible that the results obtained in this study may not be entirely applicable to programs with other breeding

1Contribution from Research Branch, Canada Department of Agriculture, and Division of Plant Industry, Australia.

2Geneticist, Division of Plant Industry, Commonwealth Scientific and Industrial Research Organization, Canberra, Australia.

3Head, Forage Crops Section, Dominion Experimental Farm, Research Branch, Canada Department of Agriculture, Swift Current, Saskatchewan. 
stock or in different environments. The results, therefore, should be regarded as a first approximation until further evidence provides a basis for future modification.

\section{MATERIALS AND METHODS}

The alfalfa population under study, consisting of 2,967 plants, has been described by Heinrichs (2) whose Table 1 gives a sample of the data. It consisted of progenies from crosses among 54 plants (of a total of 58 available) which were selected from a large population of Medicago falcata L. $x$ Medicago media Pers. origin. An occasional cross line was omitted from the analysis because there were insufficient plants or because it could not be conveniently grouped.

The data analysed were the scores for creeping root taken in the spring of 1950 when plants were approximately 2 years old. The plants were scored $1,2,3,4$, or 5 , depending on the amount of creep development $(1=$ strongly creeping rooted with a spread of 36 inches in diameter or more; $5=$ the other extreme with no creeping-rooted tendency in evidence; the other 3 scores represent degrees of creeping-root development between these extremes).

The field experiment was planted in 1948. The progeny of each cross were divided into two lots, one being planted in each of two replicates with positions assigned at random. The plants in a progeny group within a replicate were space planted adjacent to each other in a row.

The crosses were arranged in 34 groups, each group being the progeny of one plant crossed with a number of unrelated plants. The genetical relationships among plants were:

(1) Full sibs where both parents were in common

(2) Half sibs where one parent was in common

(3) Zero relationship otherwise.

The actual relationship between groups was not quite zero since in several cases groups would include one or more parents in common. Thus, plant A might be mated with $B, C, D$, and $E$, and plant $K$, with $B, F, G$, $\mathrm{H}$, and I. If $k=$ number of crosses in each of two randomly chosen half-sib groups, and $m=$ the number of crosses in one group with one parent in common with a cross in the other group, the average covariance among groups would be $\operatorname{Cov}(\mathrm{HS}) \mathrm{m} / \mathrm{k}^{2}$. Since $m$ was usually zero and seldom greater than 1 and $k$ was approximately 5, this procedure introduced a bias which would be negligible relative to other sources of statistical error.

This experimental design and genetical structure resembles that described by Kempthorne (5, Section 20.8), and the same notation will be used in the following argument, describing the common parent in a group as the sire and the variable parents as the dams. Kempthorne's population consisted of $s$ sires, each mated to a random sample of $d$ dams, the progeny of each cross being tested in $r$ replicates of a randomized block experiment. The number of individuals measured from the cross of sire $i$ with dam $i j$ in replicate $k$, was given as $N \mathrm{ijk}$, a number greater than zero for all $i$, $j$, and $k$. 


\section{RESULTS}

The modification of Kempthorne's analysis and estimation procedures made necessary because $d$ varied from sire to sire, and which takes into account a possible genotype $\mathrm{x}$ replicate interaction, has been presented by Morley (7). The modified analysis of means, and expectations of mean squares, are given in Table 1, using Kempthorne's notations in which

$\sigma_{1}{ }^{2}=$ variance among individuals within a plot, that is, within plot environmental variance plus total genotypic variance less the covariance among full sibs $(\operatorname{Cov}(\mathrm{FS}))$.

$\sigma_{\mathrm{e}}{ }^{2}=$ the variance due to plot effects plus the interaction of cross $\mathrm{x}$ replicate.

$\operatorname{Cov}(\mathrm{HS})=$ the covariance among half sibs.

$\sigma_{\mathrm{sr}^{2}}{ }^{2}=$ the interaction of sire group $\mathrm{x}$ replicate.

$\boldsymbol{\sigma}_{\mathrm{r}}{ }^{2}=$ variance among replicates.

Equating the mean squares of Table 1 to their expectations, the following estimates were obtained:

$$
\begin{array}{ll}
\sigma_{1}{ }^{2} & =1.342 \\
\sigma_{\mathrm{sr}}{ }^{2} & =(0.203-0.184) / 4.546=0.004 \text { (n.s.) } \\
\mathbf{\sigma}_{\mathrm{e}}{ }^{2} & =0.028 \text { (n.s.) }
\end{array}
$$

$\operatorname{Cov}(\mathrm{FS})-\operatorname{Cov}(\mathrm{HS})=(0.307-0.184) / 2=0.062$

$\operatorname{Cov}($ HS $)=(1.149-0.307+0.184-0.203) / 9.091=0.091$

In diploids the quantity $\operatorname{Cov}(\mathrm{FS})-2 \operatorname{Cov}(\mathrm{HS})$ provides an indication of the presence of non-additive genotypic variation. As this quantity is negative we may infer that the genotypic variation is predominantly additive. But since inheritance in alfalfa may be tetraploid, the diploid model may be inappropriate.

Following Kempthorne (4, and 5, Table 18.1) the genotypic variance in a one-locus auto-tetraploid population in which segregation is chromo-

\begin{tabular}{|c|c|c|c|c|c|c|c|c|}
\hline \multirow[b]{2}{*}{ Source } & \multirow[b]{2}{*}{ d.f. } & \multirow[b]{2}{*}{ M.S. } & \multicolumn{6}{|c|}{ E. [M.S.] } \\
\hline & & & $\sigma_{1}^{2}$ & $\sigma_{\mathrm{e}}^{2}$ & $\operatorname{Cov}(\mathrm{HS})$ & $\begin{array}{l}\operatorname{Cov}(\mathrm{FS}) \\
\operatorname{Cov}(\mathrm{HS})\end{array}$ & $\sigma \mathrm{rs}^{2}$ & $\sigma \mathrm{r}^{2}$ \\
\hline Replicates & 1 & 0.200 & 0.116 & 1 & 0 & 0 & 4.806 & 310 \\
\hline $\begin{array}{l}\text { Sires (Common } \\
\text { Parents) }\end{array}$ & 33 & 1. $149^{* * *}$ & 0.116 & 1 & 9.091 & 2 & 4.546 & 0 \\
\hline $\begin{array}{l}\text { Dams (Variable } \\
\text { Parents) in Sires }\end{array}$ & 121 & $0.307^{* *}$ & 0.116 & 1 & 0 & 2 & 0 & 0 \\
\hline Sire $\mathrm{x}$ Replicates & 33 & 0.203 & 0.116 & 1 & 0 & 0 & 4.546 & 0 \\
\hline Residual & 121 & 0.184 & 0.116 & 1 & 0 & 0 & 0 & 0 \\
\hline Within Plot* & 2657 & 1.342 & 1.000 & 0 & 0 & 0 & 0 & 0 \\
\hline
\end{tabular}
somal may be due to four kinds of genetic effect, which he termed average

TABlE 1. - ANaLysis OF VARLANCE of MEAN SCORES

*From a separate analysis (following Kempthorne) 
gene effects, viz, additive (A), dominance deviations (D), trigenic deviations $(\mathrm{T})$, and quadrigenic deviations $(\mathrm{F})$. In this paper we need to estimate the parent-offspring regression in order to study the effect on progress of various systems of selection. Using Kempthorne's definitions the following identities may be set up:

$$
\begin{aligned}
& \text { Cov Full Sib }=\frac{1}{2} A+\frac{2}{9} D+\frac{1}{12} T+\frac{1}{36} F=\operatorname{Cov}(\mathrm{FS}) \\
& \text { Cov Half Sib }=\frac{1}{4} A+\frac{1}{108} D=\operatorname{Cov}(\text { HS }) \\
& \text { Cov Parent-Offspring }=\frac{1}{2} A+\frac{1}{6} D=\operatorname{Cov}(P O)
\end{aligned}
$$

$2 \operatorname{Cov}(\mathrm{HS})$ thus provides a reasonable approximation of $\operatorname{Cov}(\mathrm{PO})$ unless dominance deviations are large. The identity $\operatorname{Cov}(\mathrm{FS})-2 \operatorname{Cov}(\mathrm{HS})$ provides an indication of the size of non-additive deviations, which in this case must be approximately zero. We may, therefore, in subsequent calculations use the relationship $\mathrm{G}=2 \operatorname{Cov}(\mathrm{FS})=2 \operatorname{Cov}(\mathrm{PO})=4 \operatorname{Cov}$ (HS) where $\mathrm{G}=$ genotypic variance (in this case all additive). The possibility exists that this relationship may change as selection changes the population, but the history of selection experiments suggests that extrapolation for five generations would not be unjustifiably venturesome.

\section{APPLICATION OF RESULTS}

The results of various kinds of selection may be predicted by using the theory developed by Dickerson and Hazel (1) and other students of animal breeding.

In material of this nature we may use mass selection alone or in combination with aids such as progeny testing. The annual improvement expected from any type of selection (9) may be written as

$$
\Delta \mathrm{g}=\sum_{\mathrm{j}} \mathrm{s} \mathrm{b}_{\mathrm{j}} \sigma \mathrm{x}_{\mathrm{j}} / \Sigma_{\mathrm{j}} \mathrm{L}_{\mathrm{j}}
$$

where $\Delta \mathrm{g}=$ the change in genotype per annum

$s_{j}=$ the selection differential in standard deviations of $x_{j}$ (calculated in the case of truncation selection of a normally distributed population as $3 / p$ where $p$ is the proportion selected, and $z$ the height of the ordinate at the point of truncation), assuming normal distribution and truncation selection.

$b_{j}=$ the regression of breeding value of the selected individual on the index of selection such as the phenotype of the plant or the mean of a progeny group. $\sigma \mathrm{x}_{\mathrm{j}}=$ the standard deviation of the index.

$L_{j}=$ the number of years from seed of one generation to seed of the next generation, that is, the generation length.

As an example we might consider a population of about 5,000 plants in which a combination of mass selection and progeny testing is to be applied. The procedure might be along the following lines:

Year 1, Spring - Plant 5,000 plants in the field.

Year 2, Spring - Score plants for degree of creep, select the best 100. Make

a number of propagules of these and plant in a polycross block for seed production. 
Year 2, Autumn - Harvest seed from the polycross block.

Year 3, Spring - Plant out progenies in a randomized block test, 50 plants per progeny.

Year 4, Spring - Score progenies, select the best 20 lines (cutting other propagules out of crossing block).

Year 4, Autumn - Harvest seed from elite crossing block for planting next spring. The cycles may then be repeated.

The proportion selected in the spring of Year 2 would be $100 / 5000=0.02$. The selection differential $S_{1}$, calculated from a table of the normal curve, would, therefore, be 2.415 standard deviations.

The regression, $b_{1}$, of the transmitting ability of the parent on its own score is $G / \sigma^{2}$, where $\sigma^{2}$ is the phenotypic variance in the original population. Taking as an estimate of $\mathrm{G}$ a function of the estimates of $\operatorname{Cov}(\mathrm{HS})$ and $\operatorname{Cov}(\mathrm{FS}), \mathrm{G}=2(0.091+0.062)=0.306$.

The appropriate value for $\sigma^{2}$ will depend somewhat on experimental layout. Assuming that all the sources of error within replicates are applicable we use the estimate

$$
\sigma^{2}=\sigma_{1}^{2}+\sigma_{\mathrm{e}}^{2}+\sigma_{\mathrm{sz}^{2}}+\frac{1}{2} \mathrm{G}
$$

$b_{1}$ is therefore

$$
\begin{aligned}
2 & \times 0.153 /(1.342+0.028+0.004+0.153) \\
& =0.306 / 1.527 \\
& =0.20
\end{aligned}
$$

The first item in the numerator of $\Delta \mathrm{g}$ is, then,

$$
\begin{aligned}
\mathrm{S}_{1} \mathrm{~b}_{1} & =2.42 \times 0.20 \times 1.236 \\
& =0.598=0.484 \sigma
\end{aligned}
$$

In calculating the effect of selection based on progeny tests allowance must be made for the reduction in genotypic variance caused by the initial selection. A modification of a formula proposed by Cochran (1) is

$$
\mathrm{G}_{\mathrm{s}} / \mathrm{G}_{\mathrm{o}}=1-\mathrm{G} / \mathrm{\sigma}^{2}[\mathrm{~S}(\mathrm{~s}-\mathrm{t})]
$$

where $G_{s}$ is the variance remaining after the selection coefficient $S$ has been obtained by truncation selection at $t$ standard deviations from the mean.

In this example:

that is

$$
\mathrm{G}_{\mathrm{s}} / \mathrm{G}_{\mathrm{o}}=1-0.20 \times 2.415(2.415-2.055)=0.83
$$

$$
\mathrm{G}_{\mathrm{s}}=0.83 \times 0.306=0.254
$$

The values for $t$ may be obtained from tables of areas and ordinates of the normal curve.

The progeny group obtained from the crossing block will consist of plants derived from a number of pollen parents, but only one seed parent in each case. Let there be $p$ progeny from each of $m$ mates providing pollen. Let the score of the $k^{\text {th }}$ offspring of the $j^{\text {th }}$ mate of the $i^{\text {th }}$ plant under test be designated $\mathrm{O}_{\mathrm{ijk}}$.

The regression of parent genotype on the mean of the progeny group is the covariance of the parent genotype and offspring mean divided by the variance of offspring means $\left(V_{\circ}\right)$. The numerator is clearly $\operatorname{Cov}(\mathrm{PO})=\mathrm{G}_{\mathrm{s}} / 2$. The denominator may be derived from the following 
model: Let $\mathrm{O}_{i j k}=\mu+\mathrm{g}_{i j k}+\mathrm{e}_{i j k}$ where $\mathrm{g}_{1 j \mathrm{k}}$ represents the genotypic value and $\mathrm{e}$ the environmental effect.

$$
\begin{aligned}
& \text { Let } E\left[g_{i j k}{ }^{2}\right]=G_{s} \\
& \mathrm{E}\left[\mathrm{e}_{\mathrm{ijk}}{ }^{2}\right]=\mathrm{E} \\
& \mathrm{E}\left[\mathrm{O}_{\mathrm{ijk}} \cdot \mathrm{O}_{\mathrm{ijk}}{ }^{\prime}\right]=\operatorname{Cov}(\mathrm{FS})=\mathrm{G}_{\mathrm{i}} / 2 \\
& E\left[O_{i j k} \cdot O_{i j}{ }^{\prime}{ }_{k}\right]=\operatorname{Cov}(H S)=G_{s} / 4 \\
& \mathrm{E}\left[\mathrm{O}_{\mathrm{ijk}}: \mathrm{O}_{\left.\mathrm{i}^{\prime}{ }^{\prime}{ }^{\prime}{ }^{\prime}\right]}\right]=\mathrm{O} \\
& E\left[\frac{\left(\mathrm{O}_{\mathrm{i}} \cdot-\mathrm{mpu}\right)^{2}}{\mathrm{mp}}\right]=\mathrm{V}_{\mathrm{o}} \\
& =\frac{1}{m^{2} p^{2}}\left[\sum_{j \mathbf{k}}\left(g_{i j \mathbf{k}}+e_{i j k}\right)\right]^{2} \\
& =\frac{1}{m^{2} p^{2}}\left[m p\left(G_{s}+E\right)+m p(p-1) \operatorname{Cov}(F S)+m p^{2}(m-1) \operatorname{Cov}(H S)\right] \\
& =\frac{1}{4 m p}\left[E+G_{s}(p(m+1)+2)\right]
\end{aligned}
$$

The improvement resulting from the second stage of selection, $S_{2} b_{2} \sigma x_{2}$ is thus calculated to be $\mathrm{S}_{2}$

$$
\begin{aligned}
& \frac{G_{s}}{2} \frac{\sqrt{V_{o}}}{V_{o}} \\
= & \frac{S_{2} G_{s} \sqrt{m p}}{\sqrt{E+G_{s}[p(m+1)+2]}} \\
= & \frac{S_{2} \times 0.254 \sqrt{m p}}{\sqrt{1.221+0.254[p(m+1)+2]}}
\end{aligned}
$$

Since 20 plants are to be selected from the 100 progeny-tested, $\mathrm{S}_{2}=1.40$.

The improvement is thus expected to be

$$
\begin{aligned}
& \frac{1.40 \times 0.254 \sqrt{\mathrm{mp}}}{\sqrt{1.729+0.254[\mathrm{p}(\mathrm{m}+1)]}} \\
& =0.634 \text { or } 0.512 \sigma \quad \text { if } \mathrm{m}=10 \text { and } \mathrm{p}=5 .
\end{aligned}
$$

The total improvement from the two stages of selection is then $(0.484+0.512) \sigma$.

Since the cycle takes 4 years the annual improvement is $0.249 \sigma$.

The effect of varying $m$ and $p$ may be calculated, with results shown in Figure 1 for $m p=48$.

The rate of improvement is near maximum if 8 to 12 mates are used but falls off fairly steeply with fewer mates than this. Since the number of mates crossing with each propagule in a crossing block is likely to be at least 4 , there seems little point in having more than 4 to 5 replicates, provided crossing is not restricted by variation in flowering time or other causes, so that only few crosses are successful. 


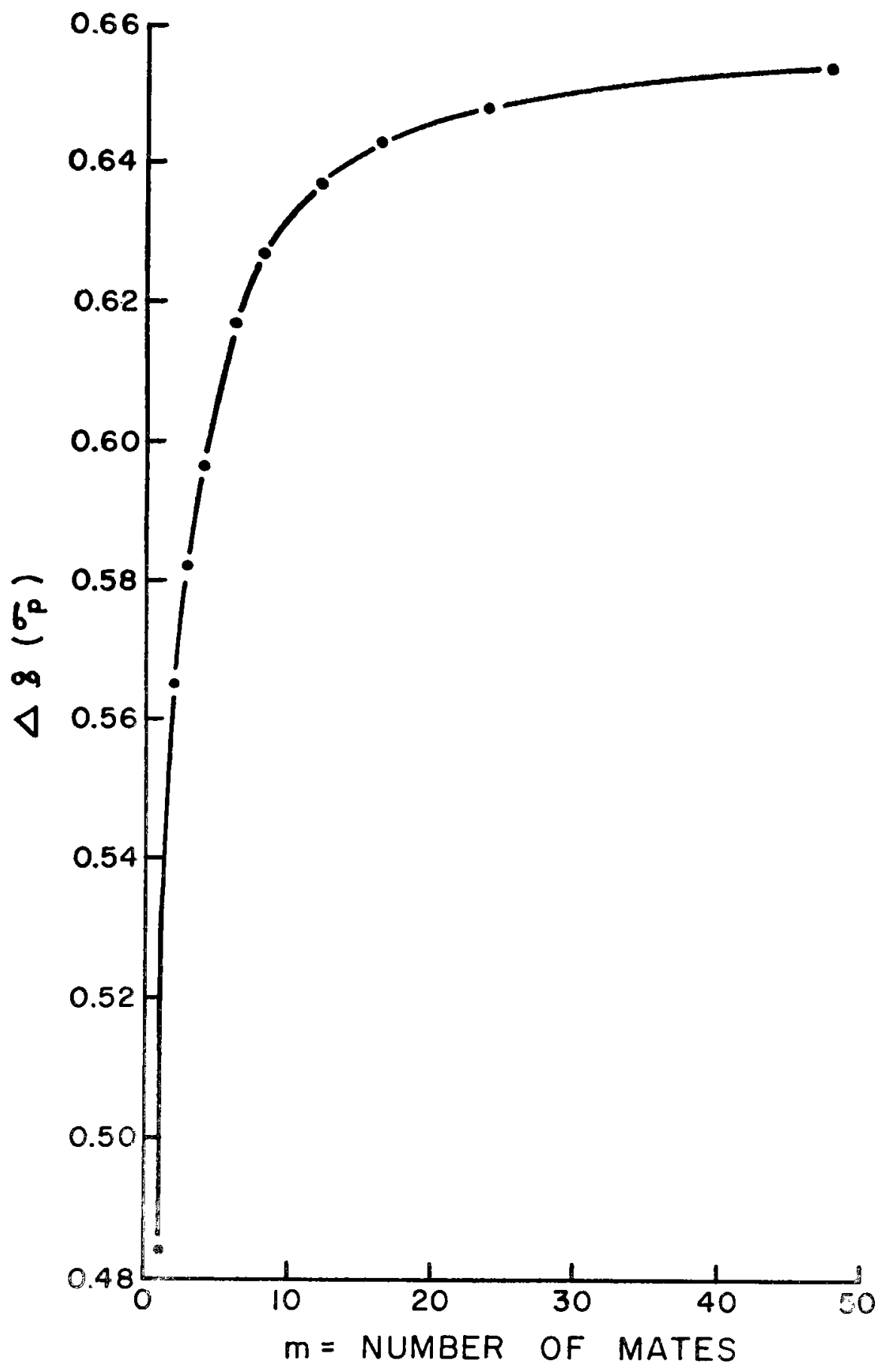

Figure 1. Effect of number of mates on efficiency of selection by progeny tests. 
The improvement from mass selection, alone or combined with progeny-testing, was predicted by using various formulae derived above, assuming always that $p$, the number of progeny per mate, was one. As can be seen from Figure 1 this result would not be affected greatly if $p$ were greater than 1 , provided $m$ were greater than 6 to 8 . The results of this examination are shown graphically in Figure 2.

The curves in Figure 2A represent the predicted annual improvement in two hypothetical populations, one of 5,000 plants, the other of 2,500 plants. These show that mass selection alone should give faster progress than mass selection combined with progeny testing, if the ratio $G / \sigma^{2}$ is 0.2 . The effect of this ratio being only 0.1 was examined in Figure 2B. Here a slight improvement is brought about by the use of progeny testing but the advantage might not be sufficient to offset the effort involved.

If progeny tests are to be used, there is little point in having more than 30 progeny per parent plant tested. In that respect the material of this study was not the most efficient design, there being generally more than 100 progeny per parent. The optimum number of progeny per plant tested seems to depend more on population size than on the ratio $G / \sigma^{2}$. In the smaller population the intensity of selection in the first stage would be less, there would be more genotypic variation among selected individuals, and hence fewer progeny would be required to select in the second stage.

In this section a fully randomized arrangement of plants has been assumed, with some device for removing replicate variance (although this was negligible in the present results). If progeny tests are set out in plots, as was done with this material, some loss of efficiency will result since the number of replications must be reduced, the replicates made larger, and comparisons less precise. In the present example replicate variation, and variation among plots within replicates, were both very small compared with genotypic differences. Therefore, the design of a test would affect precision to only a negligible extent. However, this situation may not always prevail, and the number of replicates should generally be the maximum consistent with simplicity in planting, maintenance, and recording of results.

No attempt has been made to evaluate the use of clonal testing or techniques such as that of Smith (11) of culturing root cuttings by special greenhouse techniques. A full evaluation of these must await further clarification of the correlation between such tests and field performance. Clearly any technique which shortens the generation interval is likely to be useful, but how useful cannot yet be determined.

As the level of expression increases in response to selection, progress may be increasingly hampered by the inability to discriminate between degrees of creeping. At such a stage the efficiency of selection may be aided by root culture, and also by progeny testing. Progeny testing may be especially valuable in selecting for other desired characteristics, especially if heritability is low. 


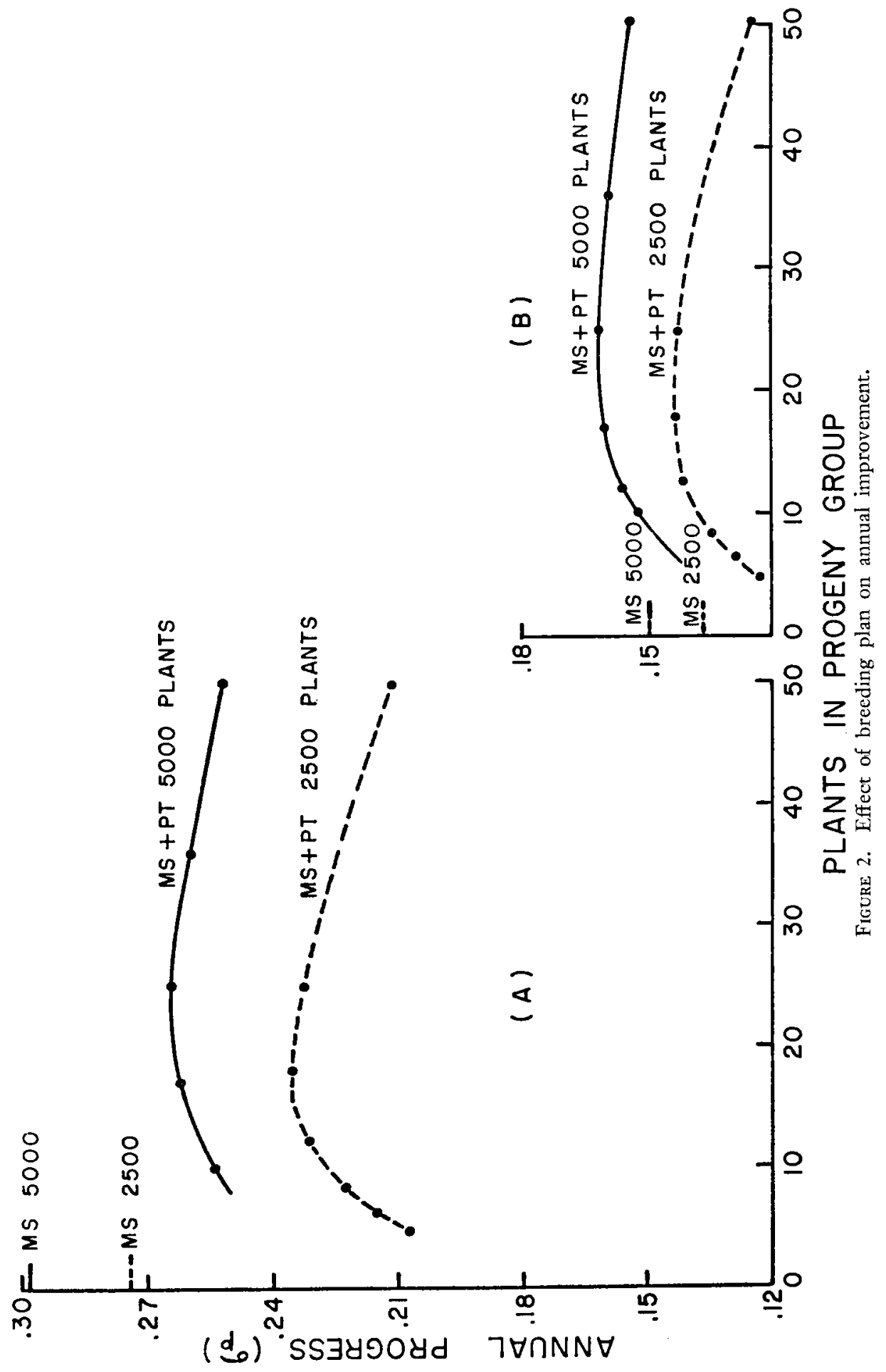




\section{CONCLUSIONS}

The genotypic variation in degree of creeping-rootedness is predominantly additive in the population studied, but was only about 20 per cent of the total phenotypic variance. Mass selection is predicted to be preferable to a combination of mass selection and progeny testing, as judged by annual improvement from selection. However, the combination may be preferred because of information required on other characters, and because the size of the genotypic variance may be continually checked. If progeny testing is used the optimum size of progeny is approximately 30 plants, provided these are obtained by crossing with at least 8 to 10 randomly selected mates. However, considerable departures from this optimum are possible without substantial loss in efficiency.

\section{ACKNOWLEDGEMENT}

The authors wish to express their sincere thanks to H. T. Fredeen, Senior Animal Husbandman, Dominion Experimental Farm, Lacombe, Alberta, for his helpful criticism of the manuscript.

\section{REFERENCES}

1. Dickerson, G. E., and L. N. Hazel. Effectiveness of selection on progeny performance as a supplement to earlier culling on livestock. J. Agr. Research 69:459-476. 1944.

2. Heinrichs, D. H. Developing creeping-rooted alfalfa for pasture. Can. J. Agr. Sci. 34:269-280. 1954.

3. Heinrichs, D. H., and J. L. Bolton. Rambler alfalfa. Can. Dept. Agr. Publ. 1030. 1958.

4. Kempthorne, $O$. The correlation between relatives in a simple autotetraploid population. Genetics 40:168-174. 1955.

5. Kempthorne, O. An introduction to genetic statistics. John Wiley \& Sons, Inc., New York, N.Y. 1957.

6. Morley, F. H. W. Selection for economic characters in Australian Merino sheep. II. Relative efficiency of certain aids to selection. Australian J. Agr. Research 3:409-418. 1952.

7. Morley, F. H. W. An effect of genotype $x$ environment interactions on estimation of genotypic covariances. Australian J. Statistics. (In Press).

8. Murray, Beatrice E. The ontogeny of adventitious stems on roots of creepingrooted alfalfa. Can. J. Botany 35:463-475. 1957.

9. Rendel, J. M., and A. Robertson. Estimation of genetic gain in milk yield by selection in a closed herd of dairy cattle. J. Genetics 50:1-8. 1950.

10. Satterthwaite, F. E. An approximate distribution of estimates of variance components. Biometrics 2:110-114. 1946.

11. Smith, D. The occurrence of adventitious shoots on severed alfalfa roots. Agronomy J. 42:398-401. 1950. 